DOI: $10.12731 / 2306-1561-2013-4-34$

\title{
CONSTRUCTING A TREE OF DATA TRANSMISSION IN WIRELESS SENSOR NETWORKS
}

Ivanova I.A., Shestakov A.A.

\begin{abstract}
This article examines the wireless sensor networks (WSN), provides a brief description of the structure of the sensor network and sensor node. Discusses graphs as models used to describe wireless sensor networks. Lists the types of network topology, which can be described by graphs. It is shown that the topology of the WSN can best be described in a random graph. Proposed to increase the duration of the network life to use a tree of data transmission, built on a limited selection of sensors of WSN. This article discusses the use of Prüfer code for organization of data transmission routes in wireless sensor networks. Algorithm of constructing the code, including supporting algorithms (for example, the algorithm of finding the leaves) is presented. Using of Prüfer code in models of wireless networks for various purposes can help in the development of the schedule of work of the WSN and to increase the overall energy efficiency of the network.
\end{abstract}

Keywords: wireless sensor networks, sensor node, random graph, tree of data transmission, Prüfer code.

\section{УДК 004.7}

\section{ПОСТРОЕНИЕ ДЕРЕВА ПЕРЕДАЧИ ДАННЫХ В БЕСПРОВОДНЫХ СЕНСОРНЫХ СЕТЯХ}

Иванова И.А., Шестаков А.А.

\section{Аннотация}

В настоящей статье рассматриваются беспроводные сенсорные сети (БСС), дается краткое описание структуры сенсорной сети и сенсорного узла. Рассматриваются графы как модели, использующиеся для описания беспроводных сенсорных сетей. Приведены типь топологии сетей, которые можно описать графами. Показано, что топология БСС наилучшим образом может быть описана случайным графом. Предложено с иелью увеличения продолжительности работь сети использовать дерево передачи данных, построенное на ограниченной выборке датчиков БСС. В настоящей статье рассматриваются вопросы использования кода Прюфера для организации маршрутов передачи данных в беспроводныхх сенсорных сетях и приведен алгоритм построения кода, включая вспомогательные алгоритмы (например, алгоритм нахождения листьев). Использование кода Прюфера в моделях 
беспроводных сетей различного назначения может помочь при разработке расписания работы элементов БСС и повысить общую энергоэффективность сети.

Ключевые слова: беспроводная сенсорная сеть, сенсорный узел, случайный граф, дерево передачи данных, код Прюфера.

\section{Введение}

Беспроводные сенсорные сети (БСС) предназначены для регистрации параметров окружающей среды в заданной области исследований. БСС построены на основе сети сенсорных узлов (СУ), каждый из которых оснащён одним или несколькими датчиками, контроллером, приемопередатчиком и источником питания [1 - 4, 14]. При этом сенсорные узлы могут быть размещены как по заранее разработанному плану, так и случайным образом, их количество может быть переменным.

СУ могут осуществлять временное хранение и предварительную обработку данных, полученных как от собственных датчиков, так и по сети от других узлов [5, 14]. Поскольку ресурсы узла (память, вычислительная мощность, емкость источника питания) крайне ограничены, и известно, что в основном энергия расходуется в процессе сбора, обработки и передачи (самая энергоемкая процедура) данных, то разрабатываются различные способы снижения нагрузки на узлы [6]. Ряд способов предусматривает использование сенсорных узлов в качестве агрегаторов и ретрансляторов данных с других СУ. В зависимости от размера сети таких уровней агрегации может быть несколько.

Для передачи данных потребителям используются, как правило, специально выделенные узлы - базовые станции (БС), выступающие в качестве шлюзов при общении с потребителями данных. БС, в отличие от СУ, не ограничена в ресурсах и используется для приёма из БСС данных, их обработки и долговременного хранения, а также является контроллером сети, организуя топологию БСС, распределение запросов к СУ для получения требующихся пользователю данных. Сенсорные узлы и базовые станции представляют собой свободную масштабируемую сеть. Базовые станции знают информацию об иерархии узлов в сети, которая регулярно обновляется, что позволяет отследить возможные изменения в расположении узлов.

C целью сохранения энергии обмен данными между узлами можно организовать по специальному расписанию. Например, можно организовать обмен информацией периодически в определённые промежутки времени. При этом в каждый момент времени работает некоторое подмножество датчиков сети, и появляется проблема построения дерева передачи данных для каждого подмножества.

В качестве математической модели для описания БСС используется теория графов. Как и в обычных проводных сетях, узлы связи представлены вершинами графа, а связи представлены ребрами. В данной работе будут рассматриваться БСС со случайными узлами. При передаче данных между узлами требуется построение дерева передачи данных, для хранения которого используется код Прюфера. 


\section{Принятые определения}

БСС рассматривается как ориентированный граф, то есть вершины соединяются направленными ребрами, при этом в графе отсутствуют петли и кратные ребра. Кроме того, при построении маршрута передачи данных от СУ к БС или команд от БС к СУ сеть рассматривается как дерево, так как передача информации по замкнутому маршруту (то есть цикл в графе) исключена.

При описании связей узлов-вершин будем пользоваться следующими понятиями [7].

Дуга - ориентированное ребро, соединяющее упорядоченную пару вершин (i, j), то есть направленное от і к j, но не наоборот.

Сильный путь, цепь - последовательность разных вершин, соединенная дугами. Например, для множества вершин $\{\mathrm{i}, \mathrm{j}, \mathrm{k}\}$ цепью является последовательность дуг $(\mathrm{i}, \mathrm{j})$, $(\mathrm{j}, \mathrm{k})$.

Слабый путь - последовательность из двух и более разных вершин, соединенных дугами с одной конечной вершиной. Например, для множества вершин $\{\mathrm{i}, \mathrm{j}, \mathrm{k}\}$ слабый путь является совокупностью дуг (i, k), (j, k).

Связность графа, представляющего БСС, описывается такими понятиями, как:

- сильно связный граф - если любая упорядоченная пара вершин может быть связана дугами;

- псевдо-сильно связный граф - если любая упорядоченная пара вершин может быть связана цепью;

- слабо связный граф - если любая упорядоченная пара вершин может быть связана слабым путем (то есть, если взять эквивалентный неориентированный граф, он будет связным).

Граф может быть разделен на подграфы. Подграф Gs - граф, множества вершин и ребер которого являются подмножествами вершин и ребер графа $\mathrm{G}$ соответственно.

Как было сказано выше, БСС рассматривается как дерево, и исходя из данных выше определений мы можем описать дерево.

Дерево - частный случай псевдо-сильно связного графа, в котором отсутствуют циклы.

Применительно к БСС будем различать:

- дерево передачи данных - дерево, в котором существует только одна вершина, не имеющая выходящей дуги;

- дерево передачи команд - дерево, в котором существует только одна вершина, не имеющая входящей дуги.

Если в составе БСС две и более БС, граф сети может быть представлен как лес, если состоит из двух и более несвязанных деревьев.

\section{Топология сети (графа)}

При исследовании БСС могут рассматриваться следующие топологии графов.

Решетка. Топология, имеющая форму двумерной сетки, на пересечении линий сетки располагаются вершины. Внутренние вершины имеют связь с четырьмя 
соседями, внешние вершины (пограничные узлы) имеют связь с тремя или двумя соседями.

Случайный граф. Представляет множество случайным образом распределенных на плоскости узлов (вершин), где каждая пара узлов связана, если эвклидово расстояние между ними не превышает определенной (заданной) величины.

Маленький мир. В такой сети не все вершины являются соседями, однако путь от одной вершины к другой возможен за некоторое небольшое количество переходов.

Безмасштабная сеть. Похоже на сеть со случайной топологией, распределение степеней вершин подчиняется степенному закону. Некоторые вершины имеют очень много связей, но большая часть имеет небольшое количество связей. Количество вершин измеряется миллионами, и для описания таких сетей используются законы статистики.

\section{Модель передачи данных в БСС}

Существуют различные модели описания графов, такие как матрица Кирхгофа, матрица смежности и другие. В тех случаях, когда речь идёт о передаче данных в БСС, то интерес могут представлять модели на основе теории перколяции $[8,9]$ и кода Прюфера.

Рассмотрим способ управления сенсорными узлами, разработанный с целью максимизации времени жизни сети. Основная идея заключается в получении выборки определенного количества датчиков, удовлетворяющих условиям, предъявляемым к зоне покрытия пользователем. Выбор этого количества датчиков основан на теории геометрической вероятности и случайной выборки при постоянной вычислительной сложности без обмена управляющей информацией с ближайшими соседями. Выбранные датчики формируют дерево передачи данных для того, чтобы избавиться от задержек при ожидании трансляции, которые имеют место при работе со случайными датчиками. Все датчики имеют одинаковую возможность отправлять собранные данные с некоторой периодичностью, так что вся исследуемая площадь покрывается с фиксированной задержкой $[10,11]$.

Процесс сбора данных со всей исследуемой области осуществляется циклически. Один цикл состоит из определенного количества сессий, в каждой из которых участвует некоторое количество сенсорных узлов, не покрывающих исследуемую область полностью. Длительность цикла, количество сессий и сенсорных узлов варьируется в зависимости от типа приложения. В этом случае процесс сбора данных можно условно назвать «приложение-зависимым». Рисунок 1 иллюстрирует «приложение-зависимый» сбор данных, основанный на компромиссе между обеспечением зоны покрытия и задержками при передаче. 


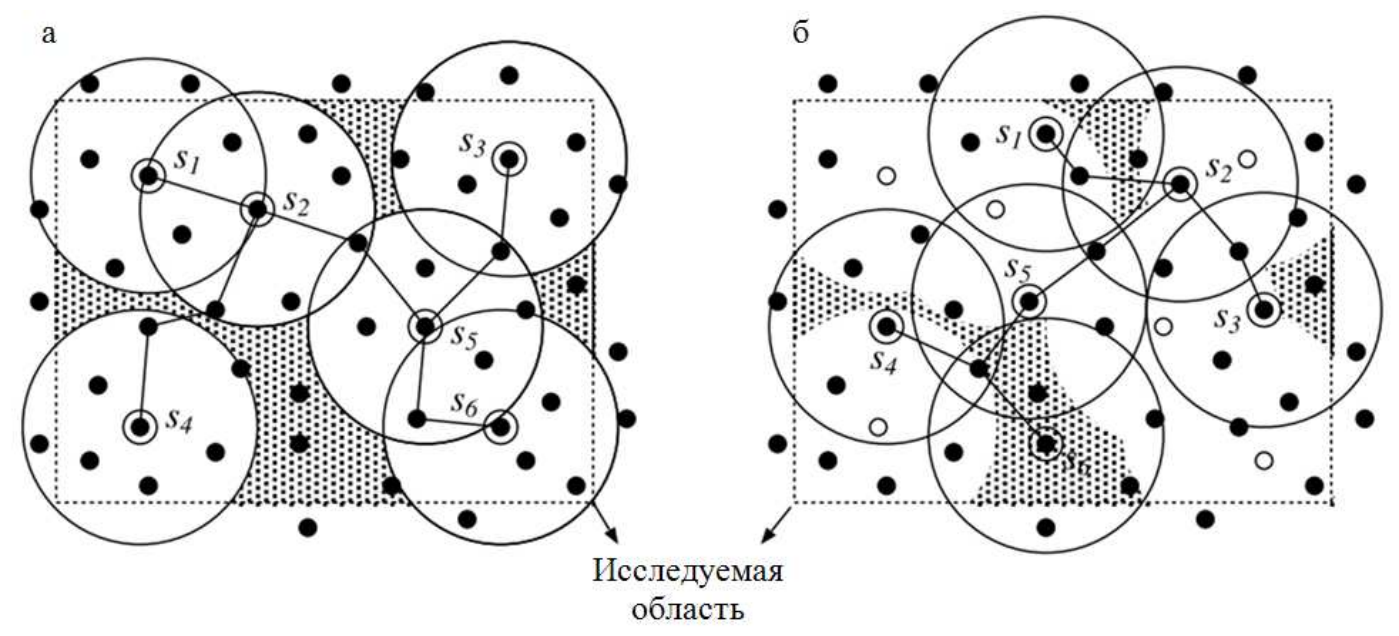

\section{Рисунок 1 - Сбор данных}

Закрашенные черные точки с маленьким кружком вокруг (s1...s6) на обоих рисунках (рисунки 1(а) и 1(б)) обозначают выбранные в настоящий момент сенсоры, обеспечивающие заданную зону покрытия (З3П), указанную пользователем или приложением, а не закрашенные точки (рисунок 1(б)) обозначают сенсоры, которые были активными в предыдущей сессии. Большая окружность обозначает зону чувствительности выбранного в данный момент сенсора. Допустим, что первое множество из шести сенсоров на рисунке 1(a) покрывает заданную часть исследуемой области, но не всю область. К примеру, закрашенная серым область на рисунке 1(a) покрывается вторым множеством датчиков, как показано на рисунке 1(б). То есть полностью исследуемая область будет покрыта после выполнения двух сессий, то есть после определенной фиксированной задержки. При такой системе сбора данных проблема заключается в том, чтобы подобрать минимально необходимое число сенсоров, удовлетворяющих 33П при каждой сессии, а $100 \%$ покрытие всей исследуемой области должно быть осуществлено за некоторое заданное время.

Рассмотрим множество сенсоров $\mathrm{N}$, расположенных на исследуемой площади Q в произвольном порядке, при этом каждый сенсор si имеет зону чувствительности SRi. B ходе текущей сессии из множества $\mathrm{N}$ выбирается минимальное число сенсоров $\mathrm{k}$ такое, что $\left(\left(\cup_{i=1}^{k} \mathrm{SRi}\right) \cap \mathrm{Q}\right) \geq 33 П$ для каждой сессии.

При выборе $\mathrm{k}$ сенсоров для каждой сессии вся исследуемая область Q будет покрыта с фиксированной задержкой Т. Пусть $\Delta \mathrm{tj}$ - продолжительность передачи данных в ходе j-й сессии при общей площади покрытия $\mathrm{SCj}=\left(\left(\mathrm{U}_{i=1}^{k} \mathrm{SRi}\right) \cap \mathrm{Q}\right) \geq 33$ П. Из этого следует, что потребуется $\delta$ последовательных сессий, таких, что

$$
\left(\left(\cup_{j=1}^{\delta} \operatorname{SRj} \cap \mathrm{Q}\right)=\mathrm{Q}\right) \wedge\left(\sum_{j=1}^{\delta} \Delta t_{j} \leq T\right) .
$$

Для решения этой проблемы применим теорию геометрической вероятности для области покрытия со случайным образом расположенными вокруг некой геометрической фигуры окружностями и технику случайной выборки $[12,13]$. 
Рассмотрим БСС как связанный граф $\mathrm{G}=(\mathrm{V}, \mathrm{E})$, где $\mathrm{V}$ - множество вершин, обозначающих сенсоры и базовые станции, служащие точками сбора данных и управляющими центрами; Е - множество ребер, обозначающих двунаправленные линии беспроводной связи между сенсорами (или сенсорами и базовыми станциями) с учетом радиуса связи. Большое количество однотипных сенсоров распределено с высокой плотностью по ограниченной площади. Они устанавливают местные связи в момент развертывания сети, а также адаптируются к изменениям топологии при выходе сенсоров из строя [12].

Рассмотрим случайные узлы, занумерованные от 1 до $\mathrm{m}$, динамические, занумерованные от 1 до d, и стационарные, занумерованные от 1 до q.

Известно, что узел $S_{\mathrm{i}}$ из $\mathrm{m}$ случайных узлов, появляющихся последовательно, имеет предпочтение установить связь с соседним узлом, номер которого $\mathrm{S}_{\mathrm{j}}$.

Каждый способ распределения узлов определяется преобразованием $\varphi$ из множества схем связи узлов $\mathrm{X}=\{1,2, \ldots, \mathrm{m}\}$.

Если $\varphi(\mathrm{i})=\mathrm{S}_{\mathrm{i}}, \mathrm{i}=1,2, \ldots, \mathrm{m}$, то вектор $\left(\mathrm{S}_{1}, \mathrm{~S}_{2}, \ldots, \mathrm{Sm}\right)$, именуемый вектором предпочтения, однозначно определяет распределения узлов предпочтения.

Рассмотрим совокупность отображений вида

$\varphi^{\prime}: \mathrm{X} \rightarrow \mathrm{Y}$,

где $\mathrm{X}=\{1,2, \ldots, \mathrm{m}\}, \mathrm{Y}=\{1,2, \ldots,(\mathrm{m}+1)\}$.

Каждое отображение $\varphi^{\prime}$ определяется производным вектором (S1',S2',..,Sm'), $\mathrm{Si}^{\prime}=\varphi^{\prime}(\mathrm{i}), \mathrm{i}=1,2, \ldots, \mathrm{m}$.

При использовании вектора предпочтения $\left(\mathrm{S}^{\prime}, \mathrm{S} 2^{\prime}, \ldots, \mathrm{Sm}{ }^{\prime}\right)$ считается, что если $\mathrm{Si}^{\prime}=\mathrm{m}+1$, то при занятом $(\mathrm{m}+1)$ динамическом узле i-й случайный узел устанавливает связь с динамическим узлом, который имеет наименьший вес.

Каждый произвольный вектор определяет расположение узлов, при котором один из узлов оказывается свободным. Общее число производных векторов равно $(\mathrm{m}+1) \mathrm{m}$.

Число возможных способов установления связи между узлами определяется следующим образом [14-17]:

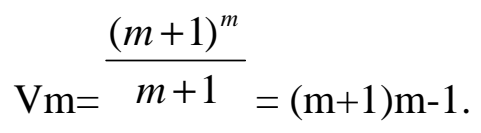

Дано отображение, которое каждому свободному дереву с $(m+1)$ вершиной ставит во взаимно однозначное соответствие вектор предпочтения. Вектору (S1,S2, .., Sm) ставится в соответствие вектор $(\pi 1, \pi 2, \ldots, \pi \mathrm{m}-1)$, где

$$
\pi \mathrm{i}=(\mathrm{Si}+1-\mathrm{Si}) \bmod (\mathrm{m}+1) \text {. }
$$

Далее вектору $(\pi 1, \pi 2, \ldots, \pi \mathrm{m}-1)$, соответствующему коду Прюфера [18-24], ставилось в соответствие дерево с $(\mathrm{m}+1)$ вершинами.

Установлено, что существует взаимно однозначное соответствие между вектором $(\pi 1, \pi 2, \ldots, \pi \mathrm{m}-1)$ и нумерованным деревом.

Определим матрицу размером $2 \times(\mathrm{n}-1)$ 


$$
\left(\begin{array}{cccc}
x_{1} & x_{2} & \ldots & x_{n-1} \\
y_{1} & y_{2} & \ldots & y_{n-1}
\end{array}\right)
$$

в которой (xi,yi) - i-ое ребро, удаленное в процессе конструирования кода Прюфера, и хі - это лист, который был удален. Последний столбец (xn-1,yn-1), где xn$1<y n-1$ будет представлять последнее оставшееся ребро. Эта матрица называется расширенным кодом Прюфера для дерева. Расширенный код Прюфера позволяет восстановить дерево передачи данных в случае потери узлов.

Существует взаимно однозначное соответствие между нумерованным деревом и кодом Прюфера, то есть однозначная схема отображения дерева в его код (из разных деревьев получается разный код) и каждому коду соответствует какое-либо дерево.

Исходное дерево легко может быть восстановлено из расширенного кода Прюфера, поскольку его ребра заданы столбцами матрицы. При этом граф, полученный из этого расширенного кода, является деревом, которое соответствует коду Прюфера, представляющему исходное дерево. Докажем это.

Возьмем множество (y1, y2, .,,yn-2). Зададим yn-1. Затем для каждого i=1,2,.,,n1 найдем хі с наименьшим номером, не принадлежащим набору $\{x 1, \ldots, x i-1\} \cup\{y i$, $\ldots, \mathrm{yn}-1\}$.

Например,

$$
\left(\begin{array}{lllll}
1 & 4 & 4 & 6 & 1
\end{array}\right) \rightarrow\left(\begin{array}{llllll}
2 & 3 & 5 & 4 & 6 & 1 \\
1 & 4 & 4 & 6 & 1 & 7
\end{array}\right) .
$$

Если наша изначальная последовательность действительно является кодом Прюфера для некоторого дерева, то можно заметить, что числа, не принадлежащие множеству $\{\mathrm{x} 1, \ldots, \mathrm{xi}-1\} \cup\{\mathrm{yi}, \ldots, \mathrm{yn}-1\}$, в действительности являются листьями этого дерева после і удалений. Так как при кодировании выбирается наименьший текущий лист, то матрица, сгенерированная выше описанным процессом, на самом деле является расширенным кодом Прюфера исходного дерева.

Теперь, взяв матрицу размера $2 \times(\mathrm{n}-1)$ с вхождениями из $\{1,2, \ldots, \mathrm{n}\}$ определим граф, чьи ребра являются столбцами матрицы. Если начать с произвольной последовательности длины n-2 из алфавита $\{1,2, \ldots, \mathrm{n}\}$ и преобразовать ее в расширенный код с помощью описанного способа, то можно утверждать, что полученный граф является деревом, более того, код Прюфера этого дерева - та самая последовательность, с которой все началось.

Для существующего кода столбцы матрицы соответствующего расширенного кода являются деревом, для которого код Прюфера является исходным кодом.

Построим граф, начиная с ребра (xn-1, yn-1) и добавляя (xn-1-i,yn-1-i) для $\mathrm{i}=1,2, \ldots, n-2$. Покажем, что получившийся граф является деревом. Действительно, начальное ребро является деревом по определению. Теперь допустим, что граф Н состоит из ребер $(\mathrm{xk}+1, \mathrm{yk}+1), \ldots,(\mathrm{xn}-1, \mathrm{yn}-1)$. Очевидно, что новый граф $\mathrm{H}^{\prime}$, полученный из Н добавлением ребра (xk, yk), также является деревом. 
Таким образом, мы можем получить процедуру для преобразования кода в дерево:

$$
\text { код } \rightarrow \text { расширенный код } \rightarrow \text { дерево. }
$$

На рисунке 2 приведен алгоритм построения дерева из кода Прюфера.

Отметим, что:

1. Если вершина v имеет степень d(v), то эта вершина встречается в коде Прюфера $\mathrm{d}(\mathrm{v})-1$ pa3.

2. Вершина со степенью 1, то есть лист, в коде никогда не встречается.

Предположим, что длины путей между всеми парами отправитель-получатель (ОП) представлены матрицей. Пары ОП обозначают листья (конечные узлы), принадлежащие дереву Т. Будем считать, что существует уникальное дерево Т, которое описывается матрицей графа между всеми его листьями. Легко показать, что если бы между какой-либо парой ОП существовал еще один путь, то у нас был бы цикл, что противоречит определению дерева. Эта идея лежит в основе взаимно однозначных отношений между матрицей графа, кодом Прюфера и нумерованным деревом.

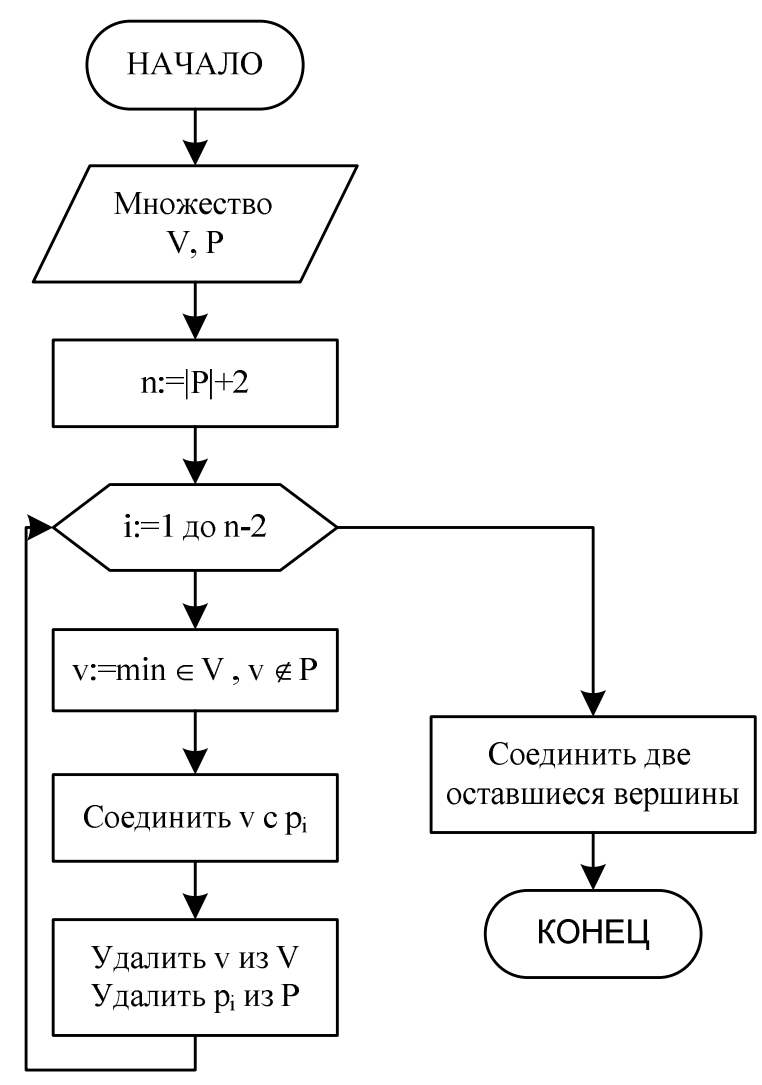

Рисунок 2 - Алгоритм построения дерева из кода Прюфера

Узлы-листья пронумерованы числами $1,2,3, \ldots, \mathrm{r}$ и составляют множество $\mathrm{R}$. Проблема состоит в том, чтобы найти правильные номера внутренних узлов, соединяющих эти листья и описать их кодом Прюфера. 
Поскольку лист с наименьшим номером легко определяется, предположим, что $\mathrm{m}$ будет первым из внутренних узлов, который соединяется с листом с наименьшей меткой. Номер $\mathrm{m}$ будет $\mathrm{r}+1$. Таким образом создадим уникальные номера в возрастающем порядке для каждого найденного внутреннего узла. Предположим, что наименьшая метка это 1. Теперь m входит в путь для всех пар ОП, которые имеют 1 в качестве отправителя или получателя, а длина пути до или от $\mathrm{m}$ уменьшается на 1 в сравнении с длиной пути до или от 1 . По алгоритму создания кода Прюфера 1 следует удалить, а его ближайшего соседа $\mathrm{m}$ добавить в код. Также вершина $\mathrm{m}$ добавляется в матрицу графа с указанием всех длин путей до связанных с ней вершин.

Теперь для т возможны два варианта:

1. m соединена с другим узлом-листом с меньшим номером (не равным 1). Это можно увидеть, проверив, существует ли путь длиной 1 между листом и $\mathrm{m}$.

2. после удаления 1 вершина т становится листом.

Это процедура повторяется для следующего наименьшего номера в R.

Когда все листья найдены, то множество вновь найденных узлов, назовем его $\mathrm{S}$, разделяется на две части: листья и внутренние узлы. Новое множество листьев R' выделяется из $\mathrm{S}$ при проверке транзитивных отношений между всеми вновь найденными узлами. Поскольку удаление листа из дерева не изменяет свойств дерева, то следующий узел с наименьшим номером в множестве $\mathrm{R}^{\prime}$ может быть легко найден. Затем для $\mathrm{R}^{\prime}$ процедура повторяется. Алгоритм останавливается, когда остается всего два узла, соединенные путем длиной 1.

Ниже приведен алгоритм построения кода Прюфера для организации схемы передачи данных.

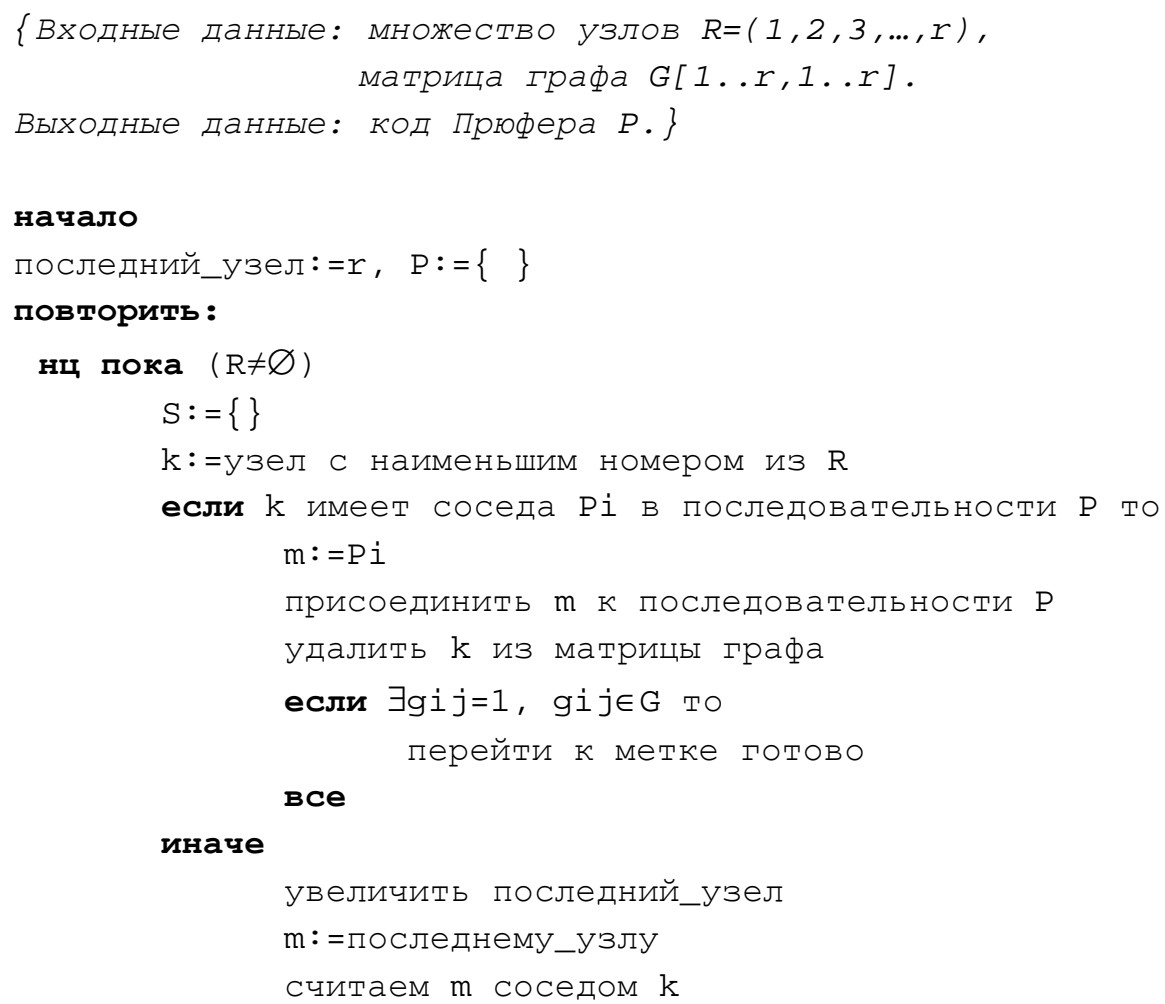




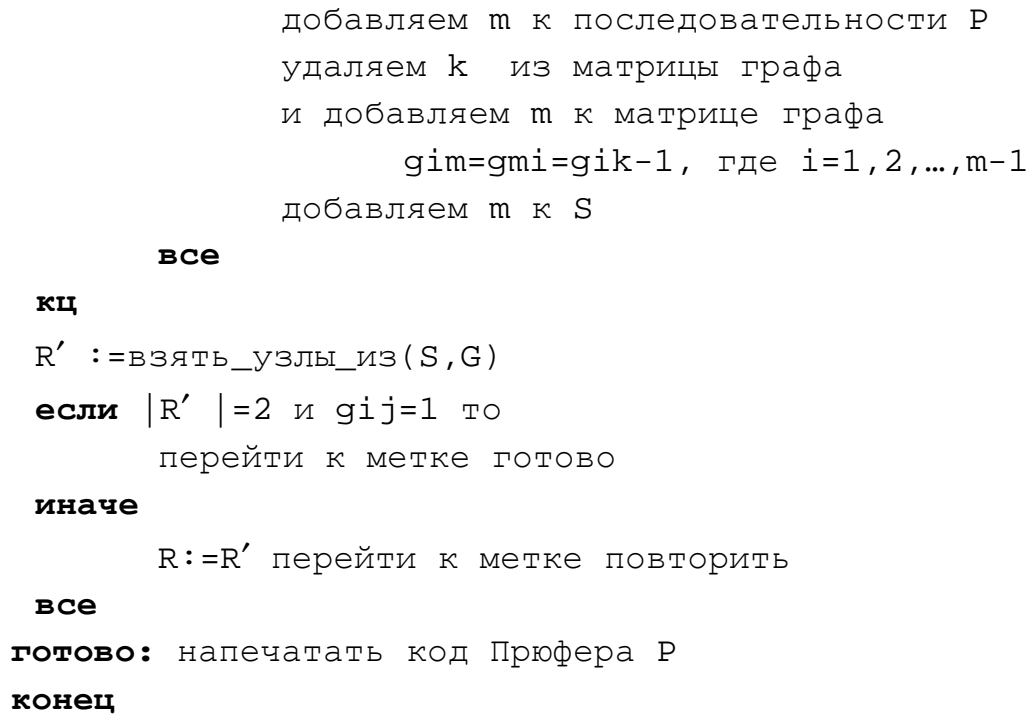

Взять_листья_из() - это процедура, отделяющая листья от вновь сформированного дерева внутренних узлов, которое было получено отбрасыванием листьев от исходного дерева. Она основана на проверке транзитивных отношений (аксиомы треугольника) между полученными внутренними узлами. Таким образом, если путь между вершинами u и $\mathrm{v}$ лежит через внутреннюю вершину w, тогда расстояние $(\mathrm{u}, \mathrm{v})=$ расстояние $(\mathrm{u}, \mathrm{w})+$ расстояние $(\mathrm{w}, \mathrm{v})$. Узел $\mathrm{m}$ рассматривается как лист, если m не встречается в качестве внутреннего узла между любой парой вершин, найденных ранее.

Далее приведен алгоритм выявления листьев.

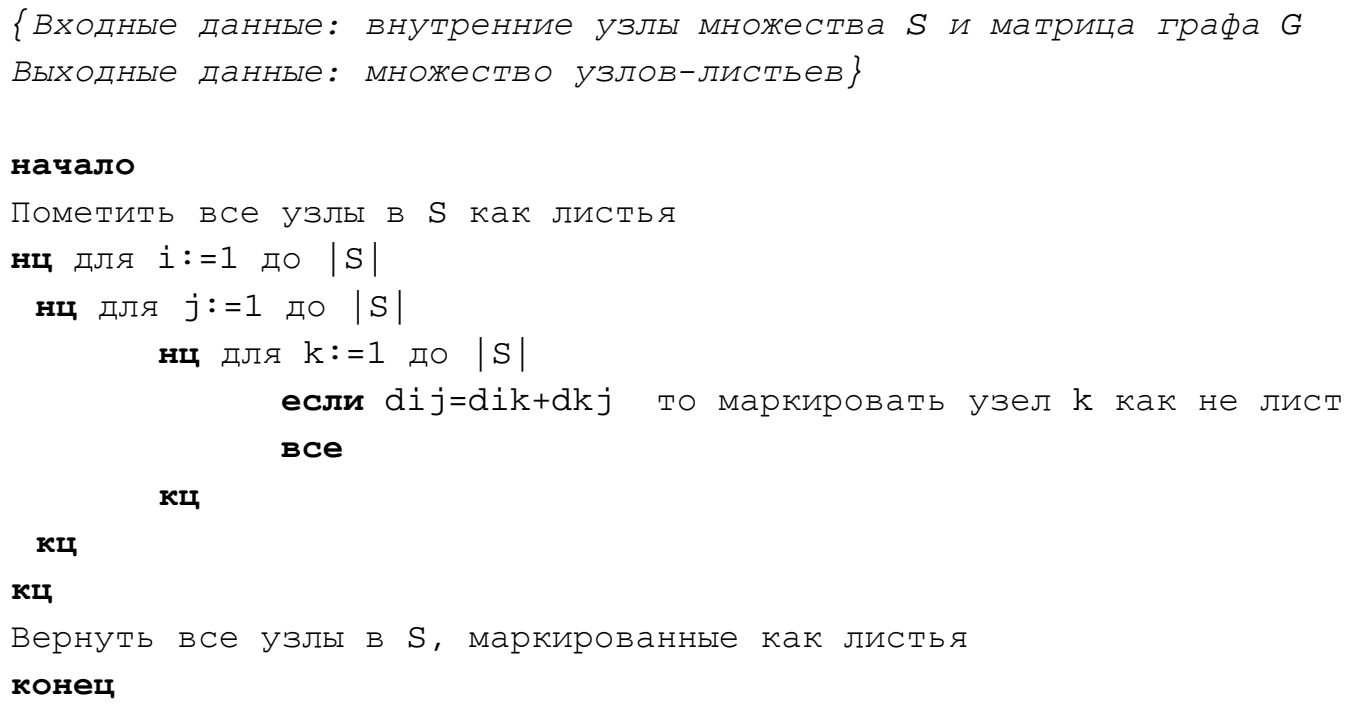

\section{Заключение}

В работе рассмотрены вопросы увеличения времени жизни беспроводной сенсорной сети. Предложено разбивать все множество датчиков БСС на подмножества, которые обеспечивали бы последовательное полное покрытие заданной площади. 
В данной работе предложена идея построения дерева передачи данных в БСС. В качестве основы для математической модели построения дерева передачи данных используется код Прюфера. Сформированное дерево передачи данных кодируется кодом Прюфера для снижения интенсивности обмена информацией между узлами, что позволит увеличить время жизни БСС.

Приведен алгоритм формирования кода Прюфера для дерева, сформированного для выборки СУ, приведены вспомогательные алгоритмы, необходимые для работы основного.

\section{Список информационных источников}

[1] Баскаков С.С. Беспроводные сенсорные сети: вопросы и ответы / Баскаков С.С. // Автоматизация в промышленности, 2008 г., №4

[2] Иванова И.А. Проблема автоматизации передачи сигнала при ограниченной мощности передатчика // Промышленные АСУ и контроллеры, 2010. № 7, с. 1516.

[3] Таненбаум Э. Компьютерные сети, СПб.: ПИТЕР, 2007, 992 с.

[4] Воронин А. Сенсорные сети [Электронный ресурс] / Воронин А. (http://www.technofresh.ru/technology/appearance/sensorable.html)

[5] Сергиевский М.В. Беспроводные сенсорные сети / Сергиевский М.В. // КомпьютерПресс, 2007, №8.

[6] Морозова Т.Ю., Никонов В.В., Тверской А.Н. Оценка качества защитного шума в беспроводных компьютерных сетях для безопасной передачи данных / Морозова Т.Ю., Никонов В.В., Тверской А.Н. // Наукоемкие технологии. 2008. Т. 9. № 7. С. $11-15$.

[7] Харари Ф., Палмер Э. Перечисление графов / Перевод с английского Г.П. Гаврилова, Москва: «Мир», 1977.

[8] Жуков Д.О., Алешкин А.С., Краевые задачи и теория перколяции в моделях обработки и передачи данных / Д.О. Жуков, А.С. Алешкин М.: Машиностроение, 2010, 213c.

[9] Антонова А.А. Среда моделирования для решения перколяционных задач / Антонова А.А., Головченко Е.Н., Петров Д.В. // Наукоемкие технологии. 2008. Т. 9. № 7. c. 26-30.

[10] Иванова И.А. Определение периметра зоны покрытия беспроводных сенсорных сетей / Иванова И.А. // Промышленные АСУ и контроллеры, 2010. № 10, с. 25-30.

[11] Y. Bejerano. Simple and efficient k-coverage verification without location information. In Proc. of IEEE InfoCom'08, Phoenix, AZ, April 2008.

[12] M. Penrose. Random Geometric Graphs. Oxford University Press, Oxford, 2003.

[13] A. Tiwari, F.L. Lewis, S.S. Ge. Wireless sensor network for machine condition based maintenance, in: Proceedings of International Conference on Control, Automation, Robotics, and Vision, 2004, pp. 461-467.

[14] Остроух А.В. Информационные технологии в научной и производственной деятельности / [ред. А.В. Остроух] - М: ООО "Техполиграфцентр", 2011. - 240 с. ISBN 978-5-94385-056-1. 